\title{
Method of Reducing the Radiation Dose to the Paediatric Age Groups in Computed Tomography
}

\author{
Farooque $\mathrm{MC}^{1}$, Ashraf Ahmed B ${ }^{2}$, Abubacker Sulaiman $\mathrm{F}^{3}$, Anand Rajamani ${ }^{4}$, Gopal Krishnan ${ }^{5}$, Divya $\mathrm{Y}^{6}$ \\ ${ }^{1}$ Final year Postgraduate, Department of Radiodiagnosis, Chettinad Hospital \& Research Institute, ${ }^{2}$ Associate Professor, \\ Department of Radiodiagnosis, Chettinad Hospital \& Research Institute, ${ }^{3}$ Professor, Department of Radiodiagnosis, Chettinad \\ hospital \& Research Institute, ${ }^{4}$ Assistant Professor, Department of Radiodiagnosis, Chettinad Hospital \& Research Institute, \\ ${ }^{5}$ Assistant Professor, Department of Radiodiagnosis, Chettinad Hospital \& Research Institute, ${ }^{6}$ Final year Postgraduate, \\ Department of Radiodiagnosis, Chettinad Hospital \& Research Institute, India
}

Corresponding author: Ashraf Ahmed B, Associate Professor, Department of Radiology, Chettinad Hospital \& Research Institute, Rajiv Gandhi Salai,Kelambakkam,Chennai,Tamilnadu -603103, India

DOI: http://dx.doi.org/10.21276/ijcmsr.2019.4.3.58

How to cite this article: Farooque MC, Ashraf Ahmed B, Abubacker Sulaiman F, Anand Rajamani, Gopal Krishnan, Divya Y. Method of reducing the radiation dose to the paediatric age groups in computed tomography. International Journal of Contemporary Medicine Surgery and Radiology. 2019;4(3):C263-C266.

\section{A B S T R A C T}

Introduction: In many clinical scenarios, Computed Tomography has a dramatic evolution which has drastically improved the diagnostic performance and enhanced its field of application. Due to its increased usage nowadays, children are at higher risk of radiation-induced cancer compared to adults which has led to increased biologic sensitivity to ionizing radiation which has resulted in more time for potential radiation-induced cancer to develop. Hence, the aim of the present study was to analyse the method among paediatrics to reduce radiation dose in computed tomography of brain.

Material and methods: The present study was a retrospective study which was conducted among pediatric population which was further subdivided in three groups based on patient age as: 0-4, 5-9, and 10-14 years. Various parameters such as tube voltage, tube current, tube rotation time, acquisition mode (sequential or helical), beam pitch (for helical CT studies), detector configuration, automated tube current modulation (enabled or disabled), CT dose index (CTDI), and doselength product (DLP) were used. For analysis of image, examination consisted of scout images plus a stack of 2.5-or 3-mmthick axial images were analysed in the study.

Results: In the present study, out of 100 subjects, 34 and 38 subjects were scanned with the help of $350 \mathrm{mAs}$ and $120 \mathrm{kVp}$ and 28 were scanned with the help of 200-300 mAs. It was found that in the age group 0-4 years; tube current time was kept at $240 \mathrm{mAs}$ and $57.9 \mathrm{kV}$ followed by $280 \mathrm{mAs}$ and $61.2 \mathrm{kV}$ for 5-9 years. For the 10-14 years age group, $310 \mathrm{mAs}$ was the tube current time followed by $82.4 \mathrm{kV}$ tube voltage settings.

Conclusion: These methods can be effective in reducing radiation dose while preserving diagnostic image quality in pediatric head CT examinations.

Keywords: Pediatric Head CT, Radiation Dose, Tube Voltage, Tube Current Time

\section{INTRODUCTION}

In 1970, due to excellent diagnostic accuracy, availability, short acquisition time, and cost-effectiveness the use of CT has increased rapidly which has led to various advances in modern medical practice. It has a growing concern for patient safety, as CT delivers increased doses of radiation than do most other diagnostic imaging procedures. ${ }^{1}$

Children and young adults are more sensitive to the stochastic effects of ionizing radiation due to their young bodies which undergoes rapid cell division. In addition, the relatively long remaining life span of children and young adults leaves ample time for expression of potential radiation effects compared with that of adults. It has been found from the literature that radiation-induced carcinogenesis is generally accepted as a stochastic process, whereas the probability of the occurrence of the effect increases with increasing radiation dose, but the severity of the effect is not influenced by the dose. ${ }^{2}$

Furthermore, children receive more effective doses due to their smaller body size, if desired pediatric CT protocols are not applied. In comparison with children hospitals, it has been found that community hospitals (where most pediatric patients are likely to undergo CT) are more likely to use adult-calibrated CT protocols. Due to these reasons, there is potential increase in cancer risk among children which is a topic of concern, although considerable amount of uncertainty exists in this matter. ${ }^{3}$

The results of several studies have suggested the potential benefits of reducing radiation dose for prevention of possible future carcinogenesis attributable to CT. In a study, it was investigated that reducing the highest $25 \%$ of doses of pediatric CT to the median level might prevent $43 \%$ of future CT-induced cancers. In a similar study, it was estimated that when the dose per CT scan was reduced by $20 \%$ and $40 \%$, 
respectively, compared with past practices in the United Kingdom; the number of future cancers potentially induced at pediatric CT was reduced by $20 \%$ and $40 \% .4,5$

Since the estimations are based on risk projection models, the actual advantage of CT dose reduction is not clear till now. However, given that results of recent epidemiologic studies on natural background and occupational protracted exposure and pediatric CT appear to be consistent with the linear non-threshold assumption, adherence to the ALARA principle is relevant and useful to save children from possible carcinogenesis caused by $\mathrm{CT}^{6}$

There are two fundamental principles for protection from medical radiation one is justification and the other is optimization. It has been suggested from these principles that CT studies should be performed only when the clinical benefit to the patient clearly exceeds potential adverse effects of radiation exposure. Every physician and radiologist must first ensure the appropriateness of the clinical indication to avoid unnecessary scans, which is the major step for reduction in overall radiation exposure to $\mathrm{CT}^{7}$

For the elimination of unnecessary radiation, the length of the scanning area on the body should also be limited to the area of concern. There are various techniques such as automated exposure control, lowering tube current and tube voltage, high-pitch acquisition, and IR algorithms which can help in reducing radiation dose or improving the image quality of low-dose pediatric CT. ${ }^{8,9}$ Hence, the aim of the present study was to analyse the method among paediatrics to reduce radiation dose in computed tomography of brain.

\section{MATERIAL AND METHODS}

This study was a retrospective study conducted among pediatric patients undergoing head CT examinations at radiologic centers in our institution. The examinations were performed in July 2018- June 2019. Every CT examinations were performed with one 64-MDCT scanner (center 1; Light Speed VCT, GE Healthcare).

All CT systems were equipped with patient size-based automated tube current modulation algorithms, as provided by each manufacturer (center 1 , Smart mA, GE Healthcare; center 2, CARE Dose, Siemens Healthcare; center 3, Sure Exposure, Toshiba Medical Systems). A total of 100 unenhanced head CT examinations of outpatient boys and girls 1 month to 14 years old were randomly selected.

The following parameters were manually retrieved from the PACS: tube voltage, tube current, tube rotation time, acquisition mode (sequential or helical), beam pitch (for helical CT studies), detector configuration, automated tube current modulation (enabled or disabled), CT dose index (CTDI), and dose-length product (DLP). Doselength product is an approximation of the energy absorbed by the patient. Subjective imaging quality was assessed by calculating the score obtained from the collected data from previous reports.

Based upon children age, the pediatric population was divided into three groups as: 0-4 years, 5-9 years and 10-14 years. It was ensured that the selected CT examination were free from artifacts (such as, those due to metallic hardware or external devices) severe enough to compromise diagnostic yield. For image analysis, the examinations consisted of scout images plus a stack of 2.5-or 3-mm-thick axial images depending on the type of scanner.

\section{STATISTICAL ANALYSIS}

The data was entered into the excel sheet and descriptive analysis was performed using statistical analysis software SPSS version 21.

\section{RESULTS}

In the present study, out of 100 subjects, 34 and 38 subjects were scanned with the help of $350 \mathrm{mAs}$ and $120 \mathrm{kVp}$ and 28 were scanned with the help of 200-300 mAs. It was found that in the age group 0-4 years; tube current time was kept at $240 \mathrm{mAs}$ and $57.9 \mathrm{kV}$ followed by $280 \mathrm{mAs}$ and $61.2 \mathrm{kV}$ for 5-9 years. For the 10-14 years age group, $310 \mathrm{mAs}$ was the tube current time followed by $82.4 \mathrm{kV}$ tube voltage settings (Table no. 1).

It was found that in the age group 0-4 years; gray matter conspicuity was kept at 0.24 and contrast to noise ratio was kept at 1.36 . For the age group, 5-9 years, 0.20 was the gray matter conspicuity followed by 1.12 contrast to noise ratio. For the 10-14 years age group, 0.18 was the gray matter conspicuity followed by 1.28 contrast to noise ratio (Table no. 2).

\begin{tabular}{|l|c|c|c|}
\hline Age & $\begin{array}{c}\text { Number } \\
\text { of } \\
\text { patients }\end{array}$ & $\begin{array}{c}\text { Tube } \\
\text { current } \\
\text { time setting } \\
\text { (mAs) }\end{array}$ & $\begin{array}{c}\text { Tube voltage } \\
\text { settings (120 kV) }\end{array}$ \\
\hline 0-4 years & 34 & 240 & 57.9 \\
\hline 5-9 years & 38 & 280 & 61.2 \\
\hline 10-14 years & 28 & 310 & 82.4 \\
\hline
\end{tabular}

Table-1: Shows the distribution of data based on tube current time and tube voltage among the children of different age groups

\begin{tabular}{|l|c|c|c|}
\hline Age & $\begin{array}{c}\text { Number of } \\
\text { patients }\end{array}$ & $\begin{array}{c}\text { Gray } \\
\text { Matter } \\
\text { Conspicuity }\end{array}$ & $\begin{array}{c}\text { Contrast-to-Noise } \\
\text { Ratio }\end{array}$ \\
\hline 0-4 years & 34 & 0.24 & 1.36 \\
\hline 5-9 years & 38 & 0.20 & 1.12 \\
\hline 10-14 years & 28 & 0.18 & 1.28 \\
\hline
\end{tabular}

Table-2: Shows the distribution of data based on Gray Matter Conspicuity and Contrast-to-Noise Ratio among the children of different age groups

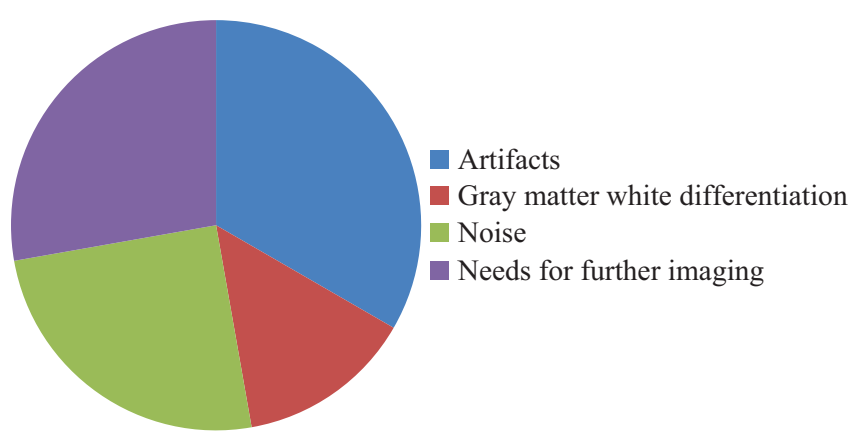

Graph-1: Shows the distribution of data based on subjective image quality 


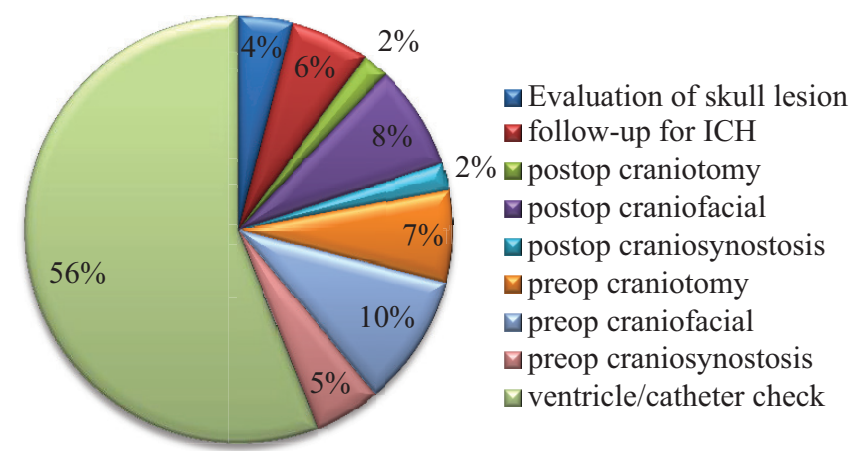

Graph-2: Shows the distribution of data based on indications and quantification of the various pathologies for low-dose head CT scans

In the present study, artifacts were found to be $24 \%$ followed by $10 \%$ gray matter white differentiation, $18 \%$ noise and $20 \%$ there was need for further imaging (Graph no. 1).

Evaluation of skull lesion was seen in 4 cases, follow-up for $\mathrm{ICH}$ in $6 \%$, post-op craniotomy in $2 \%$, postop craniofacial in $8 \%$, postop craniosynostosis among $2 \%$, preop craniotomy in $7 \%$, preop craniofacial in $10 \%$, followed by $5 \%$ in preop craniosynostosis and $56 \%$ in ventricle/catheter check (Graph no.2).

\section{DISCUSSION}

Based on approved criteria or guidelines, the first is the limiting pediatric $\mathrm{CT}$ requests to only reasonable indications and the other strategy is to adjust technical parameters on $\mathrm{CT}$ scanners to lower radiation dose while retaining diagnostic image quality. This above mentioned reduction in radiation dose was linked to a significant reduction in tube voltage and the tube current- rotation time settings used for CT examinations. ${ }^{10}$

It has been observed from the previous literature that lowered settings of tube voltage cuts radiation dose more powerfully than decreasing the tube current-time settings alone, due to absorbed dose varies by approximately the square of tube voltage and only linearly with tube current. Therefore, a reduction from $140 \mathrm{kV}$ to $120 \mathrm{kV}$ leads to a dose reduction of approximately $30 \%$, and a further $30 \%$ dose reduction can be achieved by switching from 120 to $100 \mathrm{kV} .^{11,12}$

When photoelectric effect at lower $\mathrm{x}$-ray energies was used, it helped in lowering the tube voltage settings which increased the contrast resolution owing to the higher attenuation of lower-energy $\mathrm{x}$-rays produced by lower tube voltage and $\mathrm{x}$-ray absorption. The latter effect is more pronounced with high-atomic-number objects, such as iodine from iodinated contrast material in CT angiographic studies. This increased $\mathrm{x}$-ray attenuation, however, may yield overall more tissue contrast than achieved with increased tube voltage settings, potentially improving image quality along with reduced radiation dose. ${ }^{13,14}$

In the case of unenhanced CT examinations, where identification of subtle differences in tissue attenuation may be hampered by excessively high noise levels, especially in both visual and quantitative analysis of image quality revealed greater noise in target brain areas, the use of lower tube voltage and tube current-time settings has the drawback of more image noise, which must be taken into account.

In addition, radiation dose levels in CT studies often exceed those required for diagnosis, and our finding of comparable or even higher gray matter conspicuity may be partially due to the higher intrinsic tissue contrast related to the more frequent use of lower tube voltage settings, which should partly offset the increased noise. ${ }^{15}$

The survey analysis of all of the pediatric neurosurgeons and craniofacial plastic surgeons at Seattle Children's Hospital, as well as 3 pediatric neuroradiologists, revealed that multidisciplinary support of the quality and utility of the low-dose CT scans in our selected population. These findings are in agreement with previously published smaller series that objectively demonstrated acceptable image quality, albeit with increased noise, with approximately $50 \%$ reduction in tube current. It has been a major concern in implementation of the low-dose head CT protocol which was found to be the potential for unrecognized pathology (such as hemorrhage) in the setting of reduced image quality. ${ }^{16,17,18}$

It is important to remind lastly even though a lower dose is available, low-dose CT scans still exposes each child to ionizing radiation and thus one should not expand the criteria for ordering a head CT scan. Current imaging indications must also be constantly reviewed and re-reviewed to ensure the low-dose scans are also very important. Hence, the best way to decrease the diagnostic radiation is to critically reassess the clinical utility of all radiation studies and potentially exclude imaging in children with specific indications. ${ }^{19,20}$

\section{CONCLUSION}

It was found that for unenhanced head CT examinations, the radiation dose delivered to children varied greatly. With the continuing evolution of MDCT technology and dosesaving tools, the reduction in radiation dose can be helpful in decreasing the risk associated with the CT.

\section{REFERENCES}

1. Hall EJ, Brenner DJ. Cancer risks from diagnostic radiology. Br J Radiol 2008; 81(1):362-378 5.

2. Frush DP, Donnelly LF, Rosen NS. Computed tomography and radiation risk: what pediatric health care providers should know. Pediatrics 2003; 112(5):951-957.

3. Brenner D, Elliston C, Hall E, Berdon W. Estimated risks of radiation-induced fatal cancer from pediatric CT. AJR 2001; 176(3):289-296.

4. Pearce MS, Salotti JA, Little MP, et al. Radiation exposure from CT scans in childhood and subsequent risk of leukaemia and brain tumours: a retrospective cohort study. Lancet 2012; 380(1):499-505.

5. Mathews JD, Forsythe AV, Brady Z, et al. Cancer risk in 680,000 people exposed to computed tomography scans in childhood or adolescence: data linkage study of 11 million Australians. BMJ 2013; 346(3):f2360.

6. Miglioretti DL, Johnson E, Williams A, et al. The use of computed tomography in pediatrics and the associated radiation exposure and estimated cancer risk. JAMA Pediatr 2013; 167(5):700-707.

7. Smith-Bindman R, Miglioretti DL, Johnson E, et al.Use of diagnostic imaging studies and associated radiation 
exposure for patients enrolled in large integrated health care systems, 1996-2010. JAMA 2012; 307(1):24002409.

8. Shah NB, Platt SL. ALARA: is there a cause for alarm? Reducing radiation risks from computed tomography scanning in children. Curr Opin Pediatr 2008; 20(4):243-247.

9. Pauwels EK, Bourguignon MH. Radiation dose features and solid cancer induction in pediatric computed tomography. Med Princ Pract 2012; 21(2):508-515.

10. Frush DP. CT dose and risk estimates in children. Pediatr Radiol 2011; 41(1):483-487

11. Brenner DJ. Should we be concerned about the rapid increase in CT usage? Rev Environ Health 2010; 25(2):63-68

12. Strauss KJ, Goske MJ. Estimated pediatric radiation dose during CT. Pediatr Radiol 2011; 41(suppl 2):472482.

13. Udayasankar UK, Braithwaite K, Arvaniti M, Tudorascu D, Small WC, Little S, et al: Low-dose non-enhanced head CT protocol for follow-up evaluation of children with ventriculoperitoneal shunt: reduction of radiation and effect on image quality. AJNR Am J Neuroradiol 2008; 29(4):802-806.

14. Paolicchi F, Faggioni L, Bastiani L, Molinaro S, Caramella D, Bartolozzi C. Real practice radiation dose and dosimetric impact of radiological staff training in body CT examinations. Insights Imaging 2013; 4(1):239-244.

15. Mullins ME, Lev MH, Bove $\mathrm{P}$, et al. Comparison of image quality between conventional and lowdose nonenhanced head CT. AJNR 2004; 25(2):533- 538.

16. Rosenstein M. Diagnostic reference levels for medical exposure of patients: ICRP guidance and related ICRU quantities. Health Phys 2008; 95(3):528-534.

17. Shrimpton PC, Hillier MC, Lewis MA, Dunn M. National survey of doses from CT in the UK: 2003. Br J Radiol 2006; 79(6):968-980 26.

18. International Commission on Radiological Protection. Managing patient dose in multi-detector computed tomography (MDCT). Ottawa, ON, Canada: International Commission on Radiological Protection, 2007.

19. Kalender WA, Buchenau S, Deak P, et al. Technical approaches to the optimisation of CT. Phys Med 2008; 24(1):71-79.

20. Kalra MK, Maher MM, Toth TL, et al. Strategies for CT radiation dose optimization. Radiology 2004; 230(6):619-628.

21. Waaijer A, Prokop M, Velthuis BK, Bakker CJ, de Kort GA, van Leeuwen MS. Circle of Willis at CT angiography: dose reduction and image qualityreducing tube voltage and increasing tube current settings. Radiology 2007; 242(3):832-839.

Source of Support: Nil; Conflict of Interest: None

Submitted: 20-02-2019; Accepted: 23-03-2019; Published online: 25-09-2019 